\title{
Benchmarks: An Evidence-based Approach to Creating High Performing Nurses
}

\author{
Bobbi Martin and Ronda LaVigne \\ Galen Center for Professional Development, 1031 Zorn Ave., Suite 400, Louisville 40207, KY, USA
}

\begin{abstract}
Consensus is evident among influential professional organizations (American Nurses Association, American College of Nurses, Institute of Medicine, National Council of State Boards of Nursing, World Health Organization, National Institute of Health, Quality Safety and Education for Nursing and the American Association of Critical Care Nurses) as to essential core competencies for healthcare providers. Although there is agreement among them, assessment and measurement of competence for RN's in transition is overly complex. Eleven benchmarks were developed as foundational criteria to determine readiness to practice through research of national healthcare organizations position statements on competency and subsequently cross walked with identified core competencies. This article introduces the concept of evidence-based benchmarks as a mechanism to determine readiness to practice safely, competently and independently among novice and experienced nurses who are in transition.
\end{abstract}

Key words: Transition, orientation, competency, evidence based tools, safety, practice, productive staffing.

\section{Introduction}

Nursing readiness for practice and competence is a topic that strikes at the core of quality patient outcomes and the patient's perception of care; each affecting criteria for payer reimbursement.

Assessment and measurement of competence for nurses in transition are overly complex and rely on cumbersome checkoff lists and lengthy competency performance evaluation packets. Preceptors are left to determine when a nurse in transition is ready to practice independently by their sense of readiness rather than evidence of competence.

Nurse Development Resources $^{\circledR}$ (NDR) Benchmarks are a standardized evidence based tool for competency assessment and documentation of transitioning nurses' readiness to independently manage a full patient assignment.

\section{Prevalent Transition Practices}

The immersion period refers to time spent caring for a patient assignment with a preceptor and

Corresponding author: Bobbi Martin, research fields: nursing and transition to practice. encompasses nurses in transition which include new graduates, experienced newly hired nurses, and nurses moving from one clinical area to another.

Agreement is evident among leading healthcare organizations as to essential core competencies for healthcare providers (American Nurses Association (ANA), American Association of Colleges of Nursing (AACN), Institute of Medicine (IOM), National Council of State Boards of Nursing (NCSBN), World Health Organization (WHO), National Institute of Health (NIH), Quality Safety and Education for Nursing (QSEN) and the American Association of Critical Care Nurses (AACCN)). These include patient-centered care, evidence-based practice, quality improvement, safety, teamwork, and information technology [1-8]. Although there is agreement on core competencies, ambiguity exists within nursing about methods to evaluate competence [9].

Assessment and measurement of competence for transitioning nurses is complicated. Typically, hospitals apply standard orientation times that range from four weeks for experienced nurses to 18 or more weeks for new graduate hires. However, there is no evidence to support these as reliable time-frame 
measurements of safe and competent practice. Preceptor/preceptee dyads pay little attention to the application of content contained within competency check off lists in order to complete a validation document.

Debate and controversy about methods of competency validation, diverse approaches to competency validation by hospital organizations, regulatory agency requirements, and financial constraints make standardization of competency validation among transitioning nurses untenable. As a result, the process of transitioning nurses to independent practice becomes fragmented, disorganized, and frustrating. An evidence-based tool that determines the length of the immersion period, has clear and concise criteria, and is easily used during the precepted clinical experience is a practical and sustainable solution.

\section{Competence, Competency and NDR Benchmarks}

The terms "competence" and "competency" invoke different meanings and assumptions among healthcare providers [10]. The ANA differentiates competence and competency by defining competence as the demonstration of knowledge, skills, and attributes; whereas competency is the expected criteria of performance of knowledge, skills, and attributes. In addition the ANA elaborates and states, "Competence can be evaluated by using tools that capture objective and subjective data about the individual's knowledge base and actual performance and are appropriate for the specific situation and the desired outcome of the competence evaluation" [4]. These definitions encourage nurses to engage in meaningful learning that is creative and facilitates the transfer of factual knowledge into clinical practice.

\subsection{Competency Validation}

Donna Wright's competency assessment model is gaining wide acceptance across the nation as a best practice approach to assessment and management of nurse staff competence [2, 10, 11]. Similar to the definitions provided by the ANA, her work challenges the nursing workforce to think as innovators about competency assessment. Competency evaluation is a means to assure we are providing high quality and safe patient care as opposed to surveyor and/or credentialing approval. The first step to attain a workforce with consistent performance is to clearly define competency. The following definitions provide example of the diversity that exists in nursing's definition of competency (p.7).

- The knowledge, skills, abilities, and behaviors needed to carry out a job.

- Whatever is required to do something adequately [12]

- The ability to perform a task with desirable outcomes under the varied circumstances of the real world [13]

- The effective application of knowledge and skill in the work setting [14]

Changeability in definition illuminates the need for organizations to agree on a competency definition and organize processes, policies, and competency assessments based on their definition. Equally important is deliberation and expansion of competency validation methods. Wright outlines eleven measurable and documentable approaches. Her philosophy for measuring competence embraces validation through a variety of demonstration methods but underscores best outcomes occur when verification methods align with the organizations' definition of competency. See Wright's Methods for Competency Validation Figure 1.

The next step after competencies are defined is to distinguish between initial competency assessment and ongoing competency development. Initial competency assessment begins at hire and culminates with independent practice. Initial competency assessment focuses on job knowledge, skills, and attributes necessary for independent practice during the first six months to a year in the assigned clinical setting, 


\begin{tabular}{|l|}
\hline Wright's Methods for Competency Validation \\
\hline Text/Exams \\
\hline Return Demonstrations \\
\hline Evidence of daily work \\
\hline Case studies \\
\hline Presentations \\
\hline Peer reviews \\
\hline Exemplars \\
\hline Self-Assessments \\
\hline Discussion/Reflection groups \\
\hline Mock Events/Surveys \\
\hline Quality improvement monitors \\
\hline
\end{tabular}

Fig. 1 Wright's methods for competency validation.

On-going competency assessment builds on initial assessments and reflects the ever-changing nature of the job, organization, and practice environment.

Clear differentiation between initial and ongoing competency is important to ensure the workforce is competent across the continuum. Traditionally the workforce is segmented in such a way that newly hired nurses and existing nurses follow different competency tracts. Current structures and processes complicate the end goal of creating a clearly defined competency assessment process and place arbitrary timeframes for competency validation. The use of benchmarks provides an evidence-based tool to accurately determine transition timeframes.

\subsection{Transition Timeframes}

The ability to accurately determine immersion periods is crucial for a variety of reasons: patient safety, quality patient outcomes, patient satisfaction, nurse satisfaction (preceptor and preceptee), and fiscal management of the immersion period. Presently competency assessment and validation serve as primary determinants of immersion periods-time spent caring for a patient assignment with a preceptor. Common practice uses $100 \%$ completion of assigned competencies, preceptor feedback, and an educated guess of immersion period timeframes. Few evidence-based tools exist to help organizations determine immersion timeframes $[14,15]$. There is a paucity of evidence-based tools that provide clear and concrete expectations with appropriate criteria for assessment of knowledge transfer to the clinical practice setting. Demonstration of foundational principles that develop critical thinking and problem solving are the core of Nurse Development Resources ${ }^{\circledR}$ (NDR) Benchmarks, and equip transitioning nurses with essential elements necessary to independently care for a patient assignment. Using NDR Benchmarks as a determinant of the immersion period provides an evidence-based and standardized approach that eliminates extensive orientation lengths for nurses ready to independently practice but ensures nurses who need additional time receive it.

\subsection{NDR Benchmarks}

The national conversation of how the profession transitions nurses and manages competency assessment is changing. Frameworks, criteria and tools from American Nurses Credentialing Center (ANCC) and Wright help fuel the shift by bringing context and tools to the challenge. NDR Benchmarks are part of this discussion and offer a fresh perspective and viable solution for determining nurse transition timeframes. NDR Benchmarks provide elemental criteria that assist organizations in their assessment of a transitioning nurse's readiness for safe, competent, and independent clinical practice and are intended for 
use with all nurses in transition, across all specialty practice areas, and clinical environments.

\subsubsection{Process}

A 12-month multi-state, multi-hospital beta test was conducted to implement, test, and refine NDR, which included:

- Advance ${ }^{S M}$, an interactive e-learning curriculum focused on hospital acquired conditions (HACs), core measures, and patient satisfaction with associated competencies

- Navigate $^{S M}$, a mobile web application that provides point-of-care reference for HACs and core measures along with a medication calculator

- Married State Preceptor Model (MSPM)

- Support group facilitation

- Quality improvement mentorship

Over 500 nurse leaders, preceptors, and transitioning nurses were provided workshops on how to use NDR. Early analysis of feedback from the beta test revealed a major gap in transitioning nurses to independent practice. In an effort to validate competencies, transitioning nurses failed to operationalize introductory principles necessary to manage patient care such as delegation, prioritization, and time management.

Qualitative feedback revealed preceptors spent time completing competency evaluation packets as opposed to role modeling, coaching and evaluating foundational nursing principles. This discovery served as a catalyst for further research and resulted in the development of NDR benchmarks. Benchmarks were introduced into the beta test as a determinate of readiness to practice independently of preceptor supervision. Teaching methods for all participants included onsite presentations, training videos, and monthly hosted webinars. Engage ${ }^{S M}$, an electronic management system, provided real-time progress of NDR Benchmark achievement.

\subsubsection{Development}

Research of position statements from the previously mentioned influential healthcare organizations on competency served as the NDR Benchmark underpinnings (ANA, AACN, IOM, NCSBN, WHO, NIH, QSEN, and ACCN). The research yielded specific, consistently agreed upon core competency criteria, including patient-centered care, evidence-based practice, quality improvement, safety, teamwork, and information technology. The core competencies were interwoven into eleven benchmark categories that illustrate and encompass the knowledge, skills, and behaviors necessary to manage a full patient assignment independent of a preceptor. The benchmarks eliminated reliance on complex competency evaluation packets and/or check off lists that distract preceptors and transitioning nurses from the focus of patient care coordination and management reducing fragmentation in the transition process. See Benchmark Categories Figure 2. Instead NDR Benchmark criteria provide observable actions the preceptor can assess during the precepted experience as the preceptor and transition nurse care and manage a full patient assignment together. NDR Benchmarks serve as guidelines that center the transition nurse's attention on necessary skills for safe, competent and independent management of a patient assignment.

As a bedside nurse, precepting adds an additional role with more responsibilities to an already full workload. Preceptors must learn to manage extra duties that require specific knowledge, skills, and attributes for successful outcomes. Because a bedside nurse has multiple and diverse obligations, competency assessment tools are needed that are clear, comprehensive, and easy to access. Preceptors are provided the rationale and instruction on how to use the benchmarks with the recommendation that the preceptor/preceptee dyad manage a full patient assignment together, known as the Married State Preceptor Model (MSPM) [16]. Traditionally, a patient assignment is divided between the dyad, and the transitioning nurse misses key opportunities to see critical thinking and clinical reasoning when they occur. Using benchmarks in 


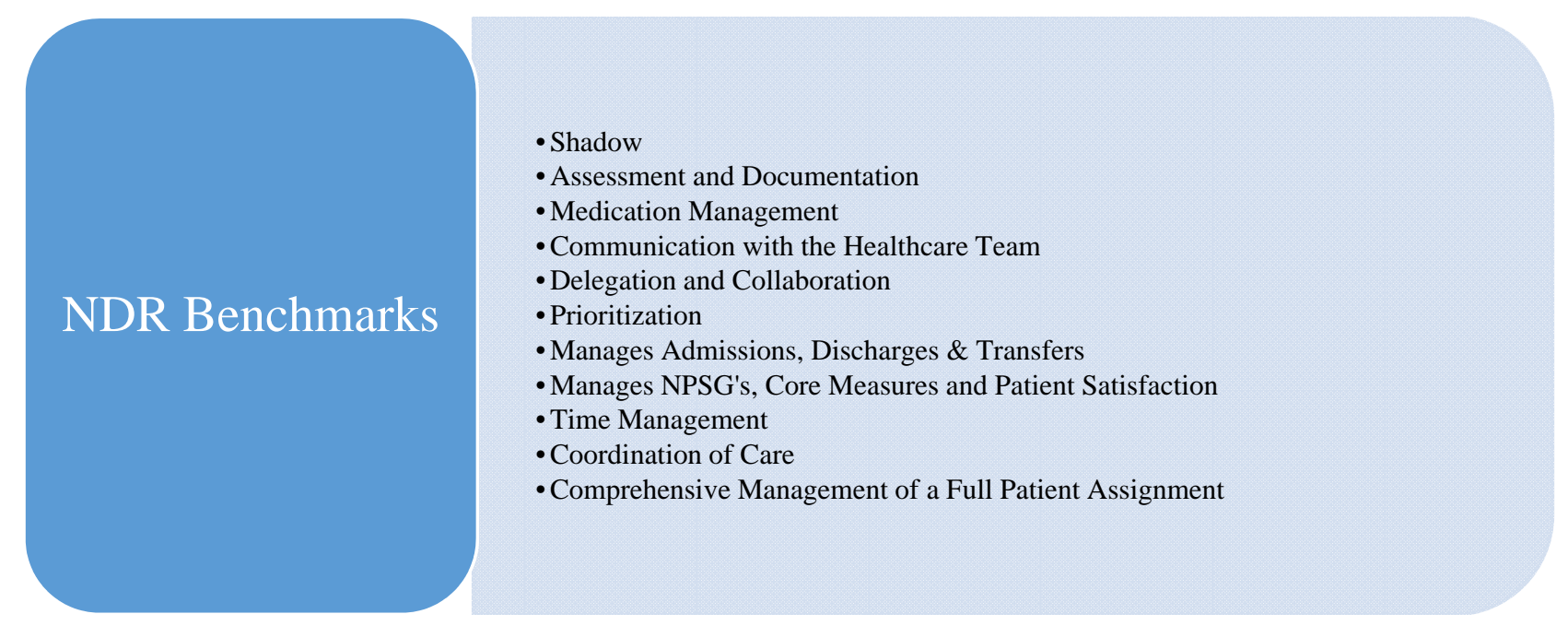

Fig. 2 Benchmark categories.

conjunction with the MSPM, the transitioning nurse begins with shadowing the preceptor and actively participates in observing professional behaviors, learning key roles and responsibilities of team members, and proper levels of delegation which are a few of the specific criteria under the shadowing benchmark. Although benchmarks are logically and theoretically ordered, nursing care delivery is complex, therefore benchmarks can be worked on simultaneously.

\subsubsection{Case Example}

It may take several weeks to achieve the medication management benchmark because it encompasses more than simply passing medications. The criteria includes administration and documentation of medications for a full patient assignment but also includes criteria to provide teaching, evaluate learning with all patients, and document patient teaching in the electronic medical record. See NDR Medication Management Benchmark Figure 3.

By virtue of the complexity of providing care to a full patient assignment, preceptors are able to assess and validate more than one benchmark at a time. In providing care using the MSPM, the preceptor is able to make multiple assessments concurrently on foundational principles.

Therefore, while the transitioning nurse is achieving the medication management benchmark, there may be opportunity to validate achievement of the communication benchmark criteria. See NDR Communication with the Healthcare Team Benchmark Figure 4.

Using the MSPM enables the preceptor to first role model then begins to assess the transition nurses progress and lastly validates achievement of benchmarks all as they care for a full patient assignment together. As the transitioning nurse progresses through and achieves benchmarks, the responsibility of the full patient assignment shifts from the preceptor to the preceptee. This transition allows the preceptor to observe and validate that the transitioning nurse can competently manage a full patient assignment independently. When all eleven benchmarks are demonstrated in clinical practice and validated by the preceptor, this marks passage of safe and competent transition to independent practice. Although benchmark completion indicates placement of the nurse into the staffing mix, validation of assigned competency sets continue. This allows for the natural occurrence of specific and repetitive learning opportunities that can be validated within their assigned competencies.

The transitioning nurse uses self-direction and autonomy to seek out unit resources that can validate competencies as experiences present in the clinical setting. This approach facilitates building peer 


\begin{tabular}{|l|}
\hline NDR Medication Management Benchmark \\
\hline Accesses resources for self and patient regarding medication actions, side effects, interactions, and teaching \\
\hline Uses informatics as needed or required to manage medications \\
\hline Maintains and communicates accurate patient medication information including reconciliation of home medications \\
\hline Administers and documents medications for full patient assignment \\
\hline Appropriately labels all medications, medication containers including syringes, tubing, medicine cups, basins, and other solutions \\
\hline Provides teaching and evaluates learning regarding medications with all patients \\
\hline Documents appropriate teaching and patient learning in the electronic medical record \\
\hline
\end{tabular}

Fig. 3 NDR medication management benchmark.

\begin{tabular}{|l|}
\hline NDR Communication with the Healthcare Team Benchmark \\
\hline Advocates for patient within the healthcare team \\
\hline Demonstrates professional interactions and communication with patients and families \\
\hline Conducts thorough handoff between shifts and care delivery locations \\
\hline Demonstrates SBAR-R communication with primary patient care providers \\
\hline Reports critical results of tests and diagnostic procedures on a timely basis \\
\hline Coordinates, consults as necessary for patients (e.g. OT/PT/RT/Dietary) \\
\hline Demonstrates ability to resolve conflict within the healthcare team including the patient, family, and interdisciplinary team \\
\hline
\end{tabular}

Fig. 4 NDR communication with the healthcare team benchmark.

relationships and promotes life-long learning. This is a paradigm shift from past practices where 100\% completion of competency sets served as the primary indicator of safe independent practice.

\section{Outcomes}

Competence is the intellect and attitude nurses must possess, competency is demonstration in practice and benchmarks are the evidence-based mechanism that safely determines the length of time necessary for nurses to practice independently. The relationship between competence, competency and NDR benchmarks becomes evident when operationalizing competency management in clinical practice. Wrights competency assessment model provides supporting rationale and the MSPM provides an effective precepting approach that enables easy use of the NDR Benchmark tool.

The impact and benefits of NDR benchmarks are trifold and include the ability for organizations to make informed strategic staffing decisions, have a standardized evidence based tool for competence assessment and documentation, and are consistent across clinical areas.

\subsection{Inform Strategic Staffing Decisions}

In light of the Institute of Medicine's recommendations of an $80 \%$ Baccalaureate-prepared nursing workforce [19], trending educational preparation data allows hospitals to hire nurses at various levels of entry thus meeting their immediate staffing needs while establishing partnerships with colleges to extend continuing education and professional development to all nurses.

Because education and experience level data is collected on each NDR user upon hire, hospitals can trend data to determine an average time needed by newly hired nurses to provide independent and safe care for a full patient assignment. For example, hospitals may hire new graduates from multiple area nursing colleges. By trending when new graduate nurses' complete benchmarks, which marks the time they are placed in productive staffing, hospitals are able to make informed hiring decisions based on evidence of actual performance. For example, nurses from X College consistently achieve benchmarks at 6 
weeks, whereas nurses from Y College consistently require 12 weeks.

This holds true for experienced nurse new hires and nurses transitioning from one unit to another. Hospitals can trend data to project the time an experienced nurse with 5 years' experience will take to be placed into productive staffing in comparison to a nurse with 2 years' experience. Or nurses from a medical/surgical unit require 8 weeks to transition to an ICU whereas nurses from labor and delivery take 12 weeks to transition to ICU. NDR benchmarks provide hospitals a tool to inform strategic staffing decisions.

\subsection{Standardize Competence Assessment \& Documentation}

Inconsistent determination of transitioning nurses' readiness to practice places patients is at risk. Standardization reduces risk and can influence positive patient outcomes. Nurse leaders, preceptors and transitioning nurses benefit from a standardized, evidence-based process. NDR Benchmarks are a solution for overcoming these hurdles and promote safe and individualized nursing transitions. Because NDR benchmarks are foundational principles that can be observed in daily management of a clinical assignment, preceptors can determine the transitioning nurses' demonstration of knowledge transfer to clinical practice. Preceptors report the concrete criteria within each benchmark which gives them measureable objectives they can discuss with their preceptee. The benchmarks provide focus for discussion and enable collaborative dialogue between the preceptor and preceptee as to how they are meeting or not meeting the criteria.

"Preceptors like the criteria for meeting the benchmarks because it gives them measureable objectives...and helps them give constructive feedback-the language they need to discuss progress with their orientee." (Quote is taken from qualitative self-reporting of beta-test site coordinator).

\subsection{Consistent Across Practice Areas}

A consistent and flexible tool is necessary for application in a variety of healthcare delivery settings. NDR benchmarks demonstrate flexible and consistence capabilities regardless of specialty areas including medical/surgical, progressive care, intensive care, emergency, labor and delivery, and pediatric departments. Discovery of this implication became evident during the beta-test, when hospitals extended benchmarks to specialty practice areas. NDR Benchmarks require transitioning nurses to demonstrate the ability to competently coordinate care for a full patient assignment and focus attention on the patient population being served.

\section{Summary}

The beta-test highlighted opportunity for vocabulary clarification and assisted with adjusting descriptions and articulation between the role of benchmarks and competency validation in clinical practice. A comprehensive but clear articulation is NDR Benchmarks incorporate top health care organizations' core competencies as the standard point of reference that outline expected levels of performance and are used to determine readiness for independent practice.

Nursing readiness for practice and competence is a topic that strikes at the core of quality patient outcomes and patient's perception of care, each affecting criteria for payer reimbursement. Organizations are challenged to demonstrate the quality of their care and competency of their nursing workforce to their community and regulatory agencies. Barriers and challenges have been related to vocabulary and methodology for competency assessment illuminating a need for evidence based measurement tools.

Prominent national organizations' core competencies outline criteria expectations but variance in methods for assessing competence in the clinical setting remains a problem in evaluating 
desired outcomes. Evidence based tools that determine readiness for independent practice is scarce. Although the ANA has better defined competence and competency, and Donna Wright provides a comprehensive model to assess and manage ongoing competencies, a gap remains as to how to determine readiness of independent practice for nurses in transition.

NDR Benchmarks are an evidence-based, predictable, and measureable tool that aids organizations with the determination of nurse readiness for independent practice. Although benchmark completion indicates placement of the nurse into the staffing mix, validation of assigned competency sets continue. This is a paradigm shift from past practices where $100 \%$ completion of competency sets served as the primary indicator of safe, independent practice. Informed strategic decisions, standardized competence assessment and documentation, and consistency across practice areas are three distinct organizational advantages to using benchmarks.

Recommendations for future research include deeper analysis of the use of benchmarks. Studies that measure the influence of benchmarks on orientation length, determinants of independent practice, and how education and experience levels influence strategic decision making will add to the national dialogue and drive high quality care.

“The nation's health depends on nursing ${ }^{\circledR}$ ” is a core belief at the Galen Center for Professional Development (GCPD). Confident the nation's health indeed depends on nursing makes the case for empowering organizations with the use of an evidence-based tool that determines a nurse's safe and competent transition to independent practice.

\section{References}

[1] American Association of Critical Care Nurses. (2015). The AACN Synergy Model for Patient Care. 2015. http://www.aacn.org/wd/certifications/content/synmodel. pcms?menu=certification. Published in 2008. Accessed
December 4, 2015.

[2] Interprofessional Education Collaborative Expert panel. Core competencies for interprofessional collaborative practice: Report of an expert panel. Washington, D.C.: Interprofessional Education Collaborative; 2011.

[3] Edlin, N. 2015. Institute of Medicine. Core Competencies Focus On Collaborative Care. Managed Healthcare Executive.

http://managedhealthcareexecutive.modernmedicine.com/ affordable-care-act/iom-core-competencies-focus-collabo rat?page=full. Published on November 21, 2013. Accessed December 4.

[4] National Council of State Boards of Nursing. (2015). Why Transition To Practice? https://www.ncsbn.org/transition-to-practice.htm. Published 2015. Accessed December 4, 2015.

[5] World Health Organization. Preparing a Health Care Workforce for the 21st Century: The Challenge of Chronic Conditions. http://www.who.int/chp/knowledge/publications/workfor ce_report.pdf Published 2005. Accessed December 4, 2015.

[6] Karic, M. 2002. "Competency and the Six Core Competencies." Journal of the Society of Laparoendoscopic Surgeons 6 (2): 95-7.

[7] Whittaker, S., Smolenski, M., and Carson, W. 2000. “Assuring Continued Competence-Policy Questions and Approaches: How should the Profession Respond?” Online Journal of Issues in Nursing 5 (3). http://www.nursingworld.org/MainMenuCategories/ANA Marketplace/ANAPeriodicals/OJIN/TableofContents/Vol ume52000/No3Sept00/ArticlePreviousTopic/ContinuedC ompetence.aspx. Published on June 30, 2000. Accessed December 4, 2015.

[8] Wright, D. 2005. The Ultimate Guide To Competency Assessment In Health Care (3rd Ed.). Minneapolis, MN: Creative Health Care Management.

[9] Ulrich, B., Krozek, C., Early, S., Ashlock, C., Africa, L., and Carman, M. 2010. "Improving Retention, Confidence, and Competence of New Graduate Nurses: Results from a 10-year Longitudinal Database.” Nursing Economics 28 (6): 363-75.

[10] Wright, D. 2013. Core Curriculum for Nursing Professional Development 4th ed. Chicago, IL: Sandra Bruce; Association for Nursing Professional Development. Chapter 21: Competency Assessment by Donna Wright.

[11] Wright, D. 2015. Competency Assessment Field Guide. A Real-World Guide for Implementation and Application. Minneapolis, MN: Creative Health Care Management.

[12] Pollock, B. 1981. "Speaking of Competencies." Health Education 12 (1): 9-13. 
[13] Benner, P. 1982. "From Novice to Expert." American Journal of Nursing 82 (3): 402-7.

[14] Del Bueno, D. 2005. "A Crisis in Critical Thinking." Nursing Education Perspectives 26 (5): 278-82.

[15] Lenburg, C., Abdur-Hahman, V., Spencer, T., Boyer, S., and Klein, C. 2011. "Implementing the COPA Model in Nursing Education and Practice Settings: Promoting Competence, Quality Care, and Patient Safety.” Nursing Education Perspectives 32 (5): 290-6.

[16] Figueroa, S., Bulos, M., Forges, E., and Judkins-Cohn, T.
2013. “Stabilizing and Retaining a Quality Nursing Work Force through the Use of the Married State Preceptorship Model.” J. Continuing Education in Nursing 44 (8): 365-73. doi: 10.3928/00220124-20130603-08.

[17] Institute of Medicine. The future of nursing: leading change, advancing health. http://books.nap.edu/openbook.php?record_id=12955\&pa ge $=\mathrm{R} 1$. Published on October 2010. Accessed December 4, 2015. 